\title{
Analysis of polycyclic aromatic hydrocarbons by supercritical fluid chromatography (SFC)
}

\author{
E. Lesellier \\ LETIAM, IUT D’Orsay, Plateau du Moulon, 91400 Orsay, France
}

\begin{abstract}
Usually, elution gradient high performance liquid chromatography using polymeric octadecyl bonded phases is chosen for this kind of separation. Due to the properties of carbon dioxide, low viscosity, high eluting power, separations obtained by supercritical fluid chromatography (SFC) are generally faster than by liquid solvents.

This paper reports the study of the PAH separation by SFC. The effects of the nature and percentage of modifiers, pressure, temperature and the choice of the stationary phase (mono or polyfunctional, high or low bonded density) are discussed. Results show that coupling two different columns is needed to reach the complete separation of the 16 PAHs requires by the standard of the Environmental Protection Agency (EPA 610). The separation is achieved in 25 minutes with a linear acetonitrile/CO $\mathrm{CO}_{2}$ mobile phase gradient.

Applied to the analysis of soil extracts, this analytical technique is enable to separate numerous other compounds than standard PAHs.
\end{abstract}

Key words. Polycyclic Aromatic Hydrocarbons (PAHs) - supercritical fluid chromatography (SFC) - stationary phases coupled columns.

\section{Introduction}

Polycyclic Aromatic Hydrocarbons (PAHs) are environmental pollutants mainly formed during the incomplete combustion of organic matter, under pyrolytic conditions [1,2]. Due to their mutagenic and carcinogenic properties, their identification and quantification in natural matrices (air, water and soils) is important.

Sixteen PAHs are classed as main pollutants by the US Environmental Protection Agency (EPA), and six of them by the Agence Francaise de Normalisation (AFNOR). Reversed phase high performance liquid chromatography (RP-HPLC) with octadecyl stationary phase is most commonly used for the determination of these weakly volatile compounds. This chromatographic method is especially convenient for the six- teen PAHs separation, because it allows to solve two different problems.

First, the PAHs mixture contains some couples of isomers, only different by configuration (phenanthrene/ anthracene or benzo(a)anthracene/chrysene), or compounds of very close molecular mass (acenaphthylene/acenaphthene) (Fig. 1).

Numerous studies have been made for determining the effects of the stationary phase characteristics on the separation of such compounds: type of bonded silica (mono or polyfunctionality), pore size, and carbon loading [3-6].

It appears that the type of bonded silica mainly controls the separation between PAH isomers. With monomeric bonded silicas (i.e. silicas prepared using monofunctional 


\section{Original articles}

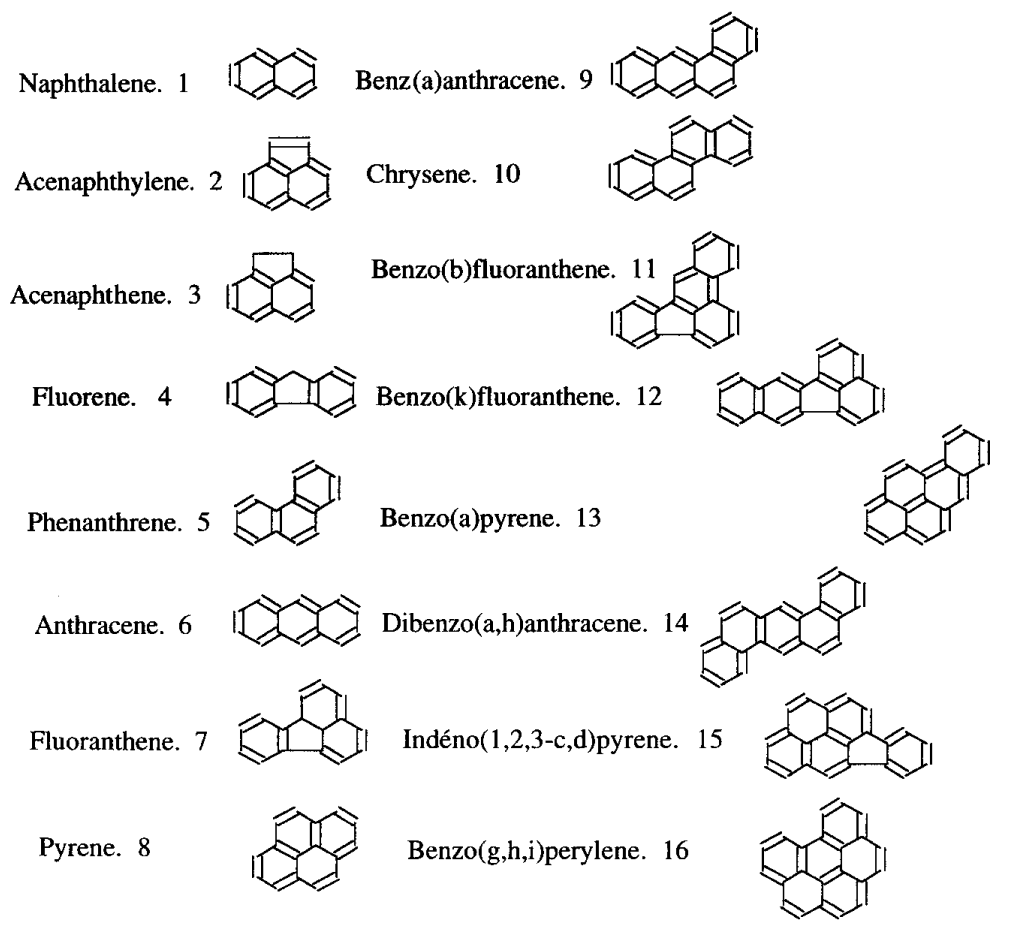

Fig. 1. Structures of the 16 PAHs of the EPA list. silanes with the exclusion of water), the separation of the PAHs is not reached: benz(a)anthracene/chrysene or benzo(k)fluoranthene/benzo(a)pyrene [3].

Conversely, polymeric octadecyl bonded silicas (i.e. those prepared using trifunctional chlorosilanes in the presence of water) have been shown to provide a good selectivity for these isomeric PAHs $[1,2,5]$.

In addition, one notices an inversion of the retention order between the two more retained compounds: benzo $(g, h, i)$ perylene and indeno(1,2,3-c,d)pyrene, that are eluted in this order using polymeric bonded silicas.

This noticeable behaviour of polyfunctional octadecyl silicas is explained by the variation of the stationary phase thickness, leading to the presence of slots into the stationary phase. The penetration of solutes into the slots is depending on their spatial configuration [5]. This three dimensional organization of the polyfunctional octadecyl bonded silica is able to discriminate the compounds through a shape recognition. For the different PAHs isomers, the calculation of the length/breadth $(L / B)$ ratio may be related to their separation capacity [3]. The elution order follows increasing $L / B$ ratios.

Secondly, the molecular mass of the sixteen PAH is quite different, ranging from two aromatic rings for naphthalene, to six rings for benzo $(g, h, i)$ perylene (Fig. 1). As the retention in RP-HPLC is depending on the ring number, the retention time of the PAHs increases strongly with the number of aromatic rings.
Isocratic analysis may be done by column-switching technique, that allows to introduce the PAHs in two identical column running with the same mobile phase while thermostated at two different temperatures [7].

Using a classical technique, an elution gradient of the mobile phase is required to achieve the separation of all the PAHs in a reasonable analytical time. However, the different gradients used are all multisptep gradients, that require a high reproducibility of the system parameters between the analysis (column, flow rate, mobile phase composition). Moreover, the equilibrium delay between two analysis increases strongly the total analysis time.

Another approach to the separation of such a complex mixture is SFC [8-10], because this technique has numerous properties, which applied to the complex mixtures fractionation allows to obtain performing separation [11].

These supercritical properties are mainly an adjustable elution power and the low viscosity of carbon dioxide used as mobile phase. For neat $\mathrm{CO}_{2}$, the supercritical state is reached at $31{ }^{\circ} \mathrm{C}$ and $7.3 \mathrm{MPa}$ in the analytical column.

The eluotropic strength of the mobile phase may be changed by varying the volumic mass of the supercritical fluid. As the supercritical $\mathrm{CO}_{2}$ is compressible, its volumic mass depends on the temperature and the outlet pressure. However, in supercritical conditions (see above) the eluotropic strength expressed by the total solubility parameter of Hildebrand, is between the alkanes and chloroform solubility parameters [12]. To increase the eluotropic strength, the addition of organic solvents, called modifiers, is required. Following this addition, changes of the fluid state 
may occur, from the supercritical state to the subcritical state. The variation of the former parameters, temperature, outlet pressure and modifier percentage have been used together in previous studies on the PAHs analysis, to decrease the retention time of the higher molecular mass compounds $[8,9]$.

The low viscosity of supercritical fluids leads to other properties of the mobile phase: high diffusion coefficient of the solutes and low pressure drop. The first one explains the great chromatographic efficiency in SFC. The second one allows to reach a more important flow rate in SFC than in HPLC, $3 \mathrm{~mL} / \mathrm{min}$ instead of $1 \mathrm{~mL} / \mathrm{min}$, which may reduce the analytical time. Due to the low pressure drop, column coupling is equally possible, either with the aim to increase the theoretical plates number (i.e. the chromatographic efficiency), using identical bonded silicas [13], or to combine different types of packing providing different selectivities [14]. This latter property seems important for reaching the complete separation of the sixteen PAHs using SFC [8].

This aspect will mainly be studied in this paper, particularly the relationship between the type of octadecyl bonded silicas and the separation of the PAH isomers in SFC. The changes in retention time due to the addition of modifiers or by the variation of temperature and outlet pressure will also be discussed.

\section{Experimental}

\section{Apparatus}

Chromatographic separations were carried out using the equipment manufactured by Jasco (Tokyo, Japan). The two pumps (Model 880-PU) were connected to a pulsation damper Sedere (Touzart et Matignon, Vitry sur Seine, France). The head of the pump used for carbon dioxide, was cooled to $-2{ }^{\circ} \mathrm{C}$ by a cryostat (Julabo F 10c, Seelbach, Germany). The pulsation damper was connected to an injection valve Model 7125 fitted with a $20 \mu \mathrm{L}$ loop (Rheodyne, Coati, CA, USA). The columns were placed in a thermostatically controlled oven (Crocosil, Cluzeau, Ste. Foy-laGrande, France).

Detection was carried out by a diode array detector (Model 910, Jasco) equipped with a high-pressure resistant cell (20 MPa). For the identification of PAHs, the spectra of PAHs have been registrered between 200 and $400 \mathrm{~nm}$, collecting one point at every $2 \mathrm{~nm}$, and collecting one spectrum every 0.8 second.

One pressure regulator 880-81 Jasco controls the outlet pressure. Chromatograms were recorded using an electronic integrator Model CR 6A (Shimadzu, Kyoto, Japan).

Some columns have been tested: A/ Hypersil ODS (250 $\times 4.6 \mathrm{~mm}$ ) (Life Sciences international, Eragny, France), B/ Kromasil C $18(250 \times 4.6 \mathrm{~mm})($ Eka Nobel, Surte, Sweden $)$, $\mathrm{C} / \mathrm{Hypersil}$ green PAH $(250 \times 4.6 \mathrm{~mm})($ Life Sciences international, Eragny, France), D/ Vydac 201 TP $54(250 \times$
$4.6 \mathrm{~mm}$ ) (Separations group, Hesperia, CA, USA), E/ Lichrospher PAH $(250 \times 4 \mathrm{~mm})$ (Merck, Darmstadt, Germany), F/ Supelcosil LC-PAH $(150 \times 4.6 \mathrm{~mm})$ (Supelco inc, Bellefonte, PA, USA).

\section{Chemicals}

Carbon dioxyde (industrial quality) was purchased from Alphagaz (Bois d'Arcy, France). Methanol and acetonitrile (HPLC grade) were purchased from Merck (Darmstadt, Germany).

The PAH mixture (EPA 610)(Supelco, Bellefonte, PA, USA) contains sixteen compounds in acetonitrile: naphthalene $\left(\mathrm{n}^{\circ} 1\right): 1 \mathrm{mg} / \mathrm{mL}$, acenaphthylene $\left(\mathrm{n}^{\circ} 2\right): 2 \mathrm{mg} / \mathrm{mL}$; acenaphthene $\left(\mathrm{n}^{\circ} 3\right): 1 \mathrm{mg} / \mathrm{mL}$, fluorene $\left(\mathrm{n}^{\circ} 4\right): 0.2 \mathrm{mg} / \mathrm{mL}$, phenanthrene $\left(\mathrm{n}^{\circ} 5\right): 0.1 \mathrm{mg} / \mathrm{mL}$, anthracene $\left(\mathrm{n}^{\circ} 6\right)$ : $0.1 \mathrm{mg} / \mathrm{mL}$, fluoranthene $\left(\mathrm{n}^{\circ} 7\right): 0.2 \mathrm{mg} / \mathrm{mL}$, pyrene $\left(\mathrm{n}^{\circ} 8\right)$ : $0.1 \mathrm{mg} / \mathrm{mL}$, benz(a)anthracene $\left(\mathrm{n}^{\circ} 9\right): 0.1 \mathrm{mg} / \mathrm{mL}$, chrysene $\left(\mathrm{n}^{\circ} 10\right): 0.1 \mathrm{mg} / \mathrm{mL}$, benzo(b)anthracene $\left(\mathrm{n}^{\circ} 11\right): 0.2 \mathrm{mg} / \mathrm{mL}$, benzo(k)fluoranthene $\left(\mathrm{n}^{\circ} 12\right): 0.1 \mathrm{mg} / \mathrm{mL}$, benzo(a)pyrene $\left(\mathrm{n}^{\circ} 13\right): \quad 0.1 \mathrm{mg} / \mathrm{mL}, \quad \operatorname{dibenzo}(\mathrm{a}, \mathrm{h})$ anthracene $\quad\left(\mathrm{n}^{\circ} 14\right)$ : $0.2 \mathrm{mg} / \mathrm{mL}$, indeno(1,2,3-c,d)pyrene $\left(\mathrm{n}^{\circ} 15\right): 0.1 \mathrm{mg} / \mathrm{mL}$, benzo $(g, h, i)$ perylene $\left(\mathrm{n}^{\circ} 16\right): 0.2 \mathrm{mg} / \mathrm{mL}$. The injection volume is $5 \mu \mathrm{L}$.

\section{Extraction of soil}

$5 \mathrm{~g}$ of industrial soil were extracted twice by sonication ( $15 \mathrm{~min}$ in $40 \mathrm{~mL}$ of methylene chloride). The two extrated fractions were collected and methylene chloride added until $100 \mathrm{~mL}$. The injection volume is $5 \mu \mathrm{L}$.

\section{Calculation}

Retention factor $k^{\prime}$ was calculated using classical equation. The void volume $\left(V_{\mathrm{o}}\right)$ was estimated from the unretained solvent peak.

\section{Results and discussions}

\section{Effect of the modification of the eluotropic strength during the analysis}

As in HPLC, an elution gradient is necessary for the analysis of PAHs by SFC.

Some specific points have been studied on the real effects of each parameter acting on the solvent strength of the supercritical fluid phase: nature and modifier content, outlet pressure and temperature.

\section{Influence of the variation in the modifier content}

Generally, using HPLC, the increase in the organic solvent content is necessary to reduce the retention time of the more retained $\mathrm{PAH}$, i.e. those which present four to six aromatic rings. The increase of modifier amount in the carbon dioxyde often allows the same retention behavior. However, different studies have shown that for some high molecular 


\section{Original articles}

mass compounds, including aromatic rings as for $\mathrm{PAH}$ (carotenoid pigments or antioxydants), the addition of polar modifiers to the neat $\mathrm{CO}_{2}$ (methanol and acetonitrile) was not followed by a decrease of the retention $(11,15-16)$, for a content in modifier ranging from 15 to $40 \%$. The use of these modifier to reduce the retention time of PAHS should be investigated.

Figure 2 shows the variation of retention of the last eluted compound, benzo $(g, h, i)$ perylene, $v s$. the percentage of modifier in carbon dioxide. This behavior is also observed for the other PAHs of the EPA 610 mixture. Fortunately, the increase of modifier content, methanol or acetonitrile, involves a decrease of the retention time. One can notice that the retention is decreased by half when the modifier is doubled from 10 to $20 \%$.

The comparison of the time factor variations reveal that the eluotropic strength is greater for the $\mathrm{CO}_{2}$ /acetonitrile mobile phase than the $\mathrm{CO}_{2} /$ methanol mobile phase. An elution gradient using acetonitrile seems to be more suitable for the separation of PAH by SFC. However, as the decrease of the experimental retention became negligible between 40 and $50 \%$ of acetonitrile in the $\mathrm{CO}_{2}, 50 \%$ will be the limit amount used.

In other respects, the analytical pressure and temperature being unchanged during the analysis, the increase of the acetonitrile content is accountable for the change of the mobile phase physical state, from a supercritical state to a subcritical one. This new state does not involve a change of the efficiency, because the theoretical plates number remains near 70000 plates per meter between 5 and $50 \%$ of acetonitrile in the mobile phase. It does not induce the separa-

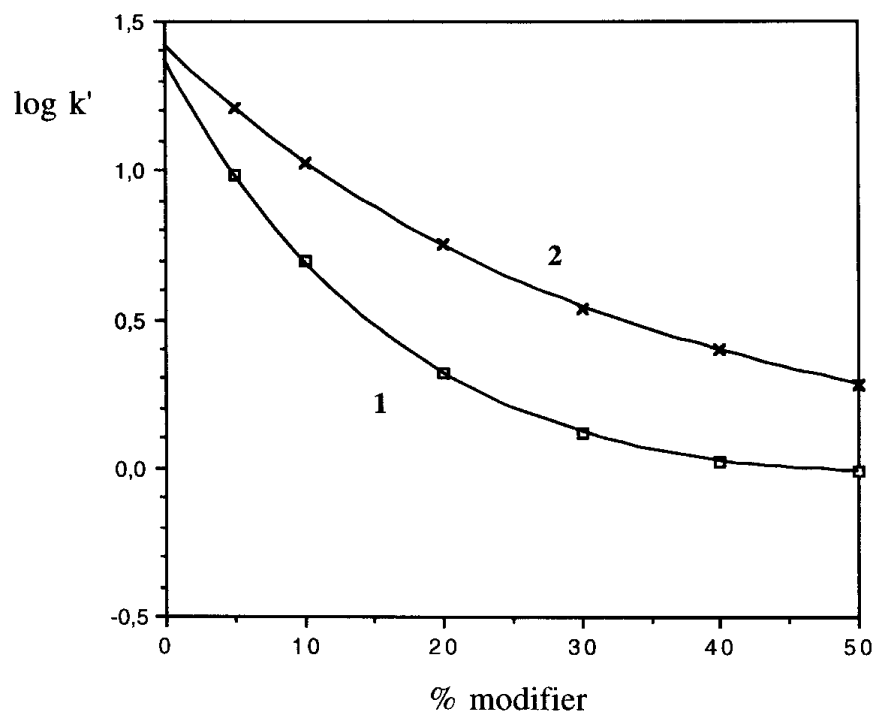

Fig. 2. Variation of $\log k^{\prime}$ of the $\operatorname{benzo}(g, h, i)$ perylene $v s$. the modifier content in the $\mathrm{CO}_{2}(\mathrm{v} / \mathrm{v})$. 1/ acetonitrile; 2/ methanol. Column: Hypersil ODS. tion of the subcritical fluid into liquid and gazeous phases, as can be proved by the stability of the UV baseline of the detector.

\section{Effect of temperature and pressure outlet}

The eluotropic strength of sub or supercritical fluid may also be modified changing the density of the fluid.

Because variations of temperature and outlet pressure act on the density, these parameters may be used to decrease the retention time of strongly retained compounds.

However, the changes of these chromatographic parameters set experimental and theoretical problems.

First, complex equipment is required to achieve these variations. After the analysis, the come back to the initial temperature is time-consuming and became a restrictive step.

Secondly, a change of these parameters is also able to modify selectivity in an opposite way to the modifier gradient. This double variation of selectivity may reduce the separation and may be difficult both to understand and to reproduce.

However, to determine if an increase of temperature can strongly reduce the retention time, a study has been done with mono and polyfunctional bonded silicas. The results show that increasing twice the temperature decrease only the retention time of $10 \%$. As this reduction is weak in comparison to that obtained by an increase in the modifier content, the change of temperature during the analysis was forsaken.

Other studies have shown that the effect of pressure on the retention time may be different following the modifier content $[11,17]$. For high molecular weight compounds such as triacylglycerols, when using a mobile phase with a high modifier percentage, the increase of the outlet pressure is not necessarily followed by a decrease of the retention time. This behavior is due to the low mobile phase compressibility in the presence of high modifier content in the carbon dioxide. Nevertheless, two analyses using the same composition gradient of mobile phase have been done, at two different outlet pressures: 10 and $15 \mathrm{MPa}$ (Fig. 3).

The results underline an effect of the outlet pressure on the retention time which depends on the molecular mass of the PAHs. The smaller the molecular mass of the compound, the greater the retention variation of the PAHs. The variation is equal to $6.5 \%$ for fluorene and only to $0.6 \%$ for benzo $(g, h, i)$ perylene.

As the smaller PAH are eluted quickly, the mobile phase in which they are solubilised is rather rich in carbon dioxide. In this case, the increase of outlet pressure changes the density and the solvent strength of the sub/supercritical fluid, because of the compressibility of this high carbon dioxyde content mobile phase. Thus, the variation of retention is measurable. 
$\%$ of retention variation

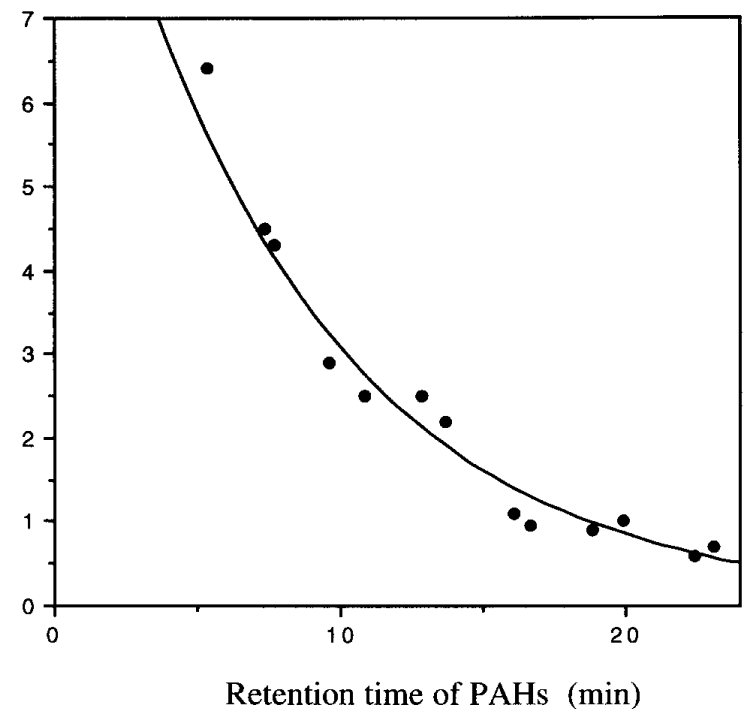

Fig. 3. Percentage of variation of the retention time of the 16 PAHs by changing the outlet pressure (from 10 to $15 \mathrm{MPa}$ ) vs. an the retention time obtained with an outlet pressure equal to $10 \mathrm{MPa}$. Column: Kromasil C18; mobile phase: elution gradient from $\mathrm{CO}_{2} / \mathrm{ACN}(100: 0 ; \mathrm{v} / \mathrm{v})$ to $\mathrm{CO}_{2} / \mathrm{ACN}(60: 40 ; \mathrm{v} / \mathrm{v})$ in 20 minutes.

When the greatest PAHs are eluted from the column, the modifier content of the mobile phase is higher than for small PAHs. Then, the compressibility of the mobile phase is strongly reduced, because the addition of modifier increase also the mobile phase viscosity and the internal pressure. Consequently, the retention variation for the great $\mathrm{PAH}$ is negligible when changing the outlet pressure.

As a result, only the change of the modifier content during the analysis has been selected to reduce the analytical time of the PAHs.

\section{Influence of the stationary phase on the separation}

Six Octadecyl bonded silicas have been tested with gradient condition.

These columns are polyfunctional bonded silicas (Hypersil Green PAH; LiChrospher PAH; Vydac 201 TP 54, Supelcosil LC-PAH), or monofunctional bonded silicas (Kromasil C18; Hypersil ODS) [4,5,11].

These characteristics (kind of functionality, linkage density) have been estimated by a SFC test, which allows to estimate the selectivity between the cis/trans isomers of $\beta$ carotene [11]. This test provides the same results as the Nist 689 test done by Sander and Wise [5], but may be less timeconsuming and requires just one injection of a $\beta$-carotene isomers mixture obtained by laying a $\beta$-carotene solution exposed to daylight.
The cis/trans selectivity values indicates the nature of the stationary phase: higher than 1.2 for polyfunctional bonded silicas (1.26 for Vydac 201 TP 54), between 1.1 and 1.2 for monofunctional bonded silicas with a high bonded density (1.13 for Kromasil C 18), and between 1 and 1.1 for monofunctional bonded silica with a low bonded density (1.06 for Hypersil ODS).

The sixteen PAHs of the EPA 610 mixture have been chromatographied with the same analytical conditions, using the six columns (Fig. 4). None of the columns allows to achieve the separation of the sixteen PAH. Four columns give similar results: Hypersil Green PAH, LiChrospher PAH, Supelcosil LC-PAH, and Vydac 201 TP 54. With these polyfunctional bonded silicas, two coelutions are noticed: acenaphthylene/acenaphthene (2/3), and indeno(1,2,3c,d)pyrene/benzo $(g, h, i)$ perylene $(15 / 16)$. A begin of separation for the last couple appears on the LiChrospher $\mathrm{PAH}$, with the same elution order than the one observed in HPLC (benzo $(g, h, i)$ perylene/indeno( $1,2,3-\mathrm{c}, \mathrm{d})$ pyrene).

Using the low density monofunctional bonded silica (Hypersil ODS), more compound couples are unresolved: acenaphthene/fluorene (3/4); phenanthrene/anthracene (5/6), benzo(b)fluoranthene/benzo(k)fluoranthene (11/12).

The high density monofunctional bonded silica provides intermediate results (Kromasil C18), while only the benzo(b)fluoranthene/benzo(k)fluoranthene couple (11/12) is not separated.

Thus, like the behaviors reported in HPLC, the separation of the PAHs in sub/supercritical fluid chromatography is, in part, depending on the tridimensional structure of the stationary phase. However, if this phenomenon allows to explain the separation difference for the indeno(1,2,3c,d)pyrene/benzo $(g, h, i)$ perylene couple $(15 / 16)$, it cannot be applied for understanding why two other couples of small PAHs are not separated using low density monofunctional bonded phase.

\section{Development of the final separation}

Because one column does not allow the separation of the sixteen PAHs, two columns have to be associated, each one providing its own selectivity.

Moreover, this column association increases the efficiency of separation $(N)$, while the retention factor $\left(k^{\prime}\right)$ is quite constant. In this case, the resolution is mainly depending on the selectivity changes. It explain why a window diagram of selectivity is used to investigate the changes in separation instead of a resolution measurments.

Figure 5 shows the variation of selectivity for different unresolved couples of PAH vs. the cis/trans $\beta$-carotene selectivity that determines the functionality and the density of the bonded phase [11]. It underlines that replacing a monofunctional octadecyl bonded phase by a polyfunctional one increases the separation of the couples of PAH: 4/3; 6/5 and $12 / 11$, and at the opposite, decreases these of the couples $3 / 2$ and $15 / 16$. 


\section{Original articles}
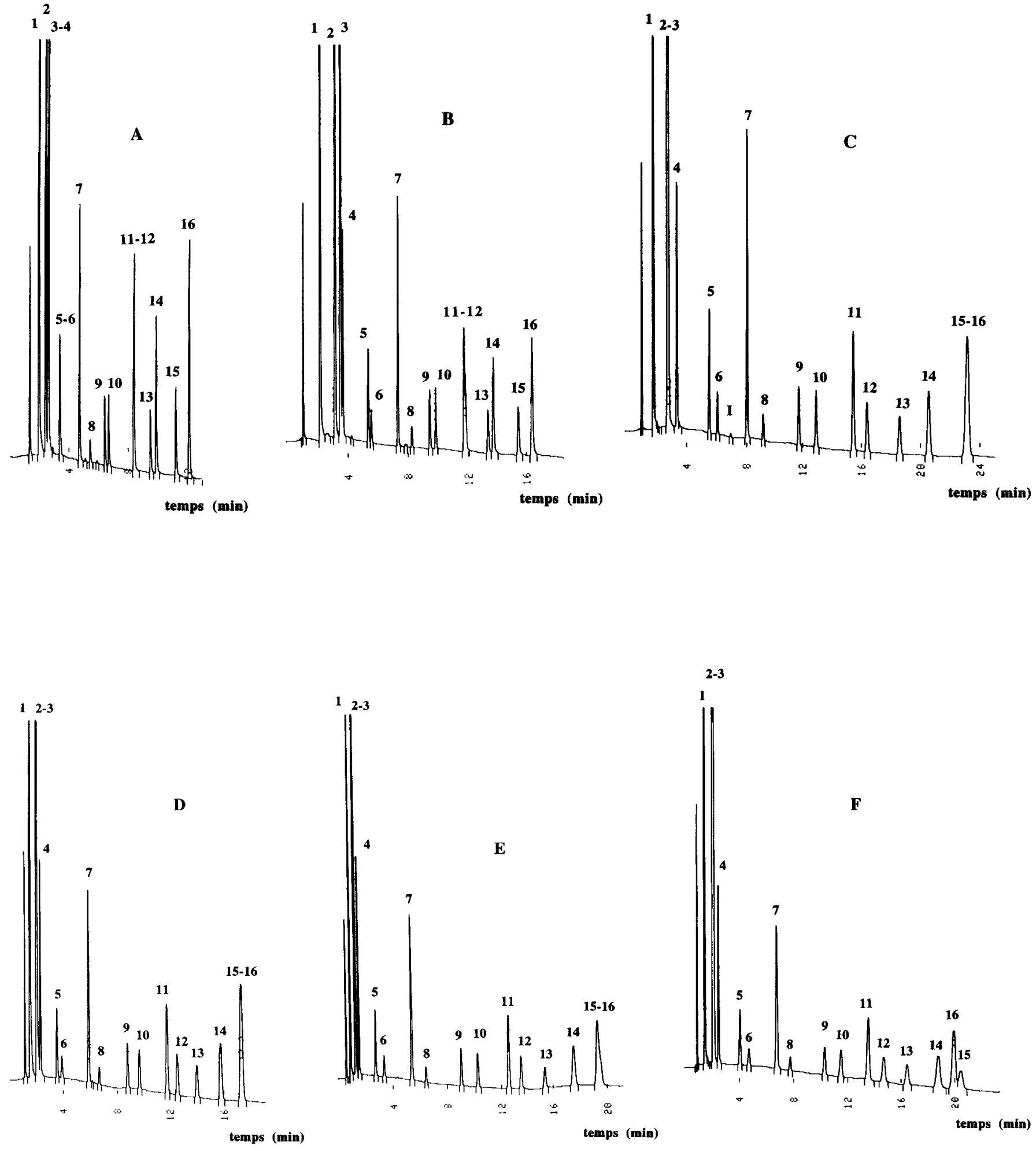

Fig. 4. Chromatogram of the $16 \mathrm{PAHs}$ using different columns. Mobile phase: elution gradient from $\mathrm{CO}_{2} / \mathrm{ACN}(100: 0$; $/ \mathrm{v})$ to $\mathrm{CO}_{2} / \mathrm{ACN}$ $(60: 40 ; \mathrm{v} / \mathrm{v})$ in 20 minutes, then $\mathrm{CO}_{2} / \mathrm{ACN}(60: 40 ; \mathrm{v} / \mathrm{v})$ during 5 minutes. Total duration 25 minutes. $T=40{ }^{\circ} \mathrm{C} ;$ Outlet pressure $=$ $10 \mathrm{MPa}$; flow rate $=3 \mathrm{~mL} / \mathrm{min}$; UV detection: $210 \mathrm{~nm}$. A/ Hypersil ODS; B/ Kromasil C18; C/ Hypersil Green PAH; D/ Vydac 201 TP 54; E/ Supelcosil LC-PAH; F/ LiChrospher PAH. 


\section{PAHs selectivity}

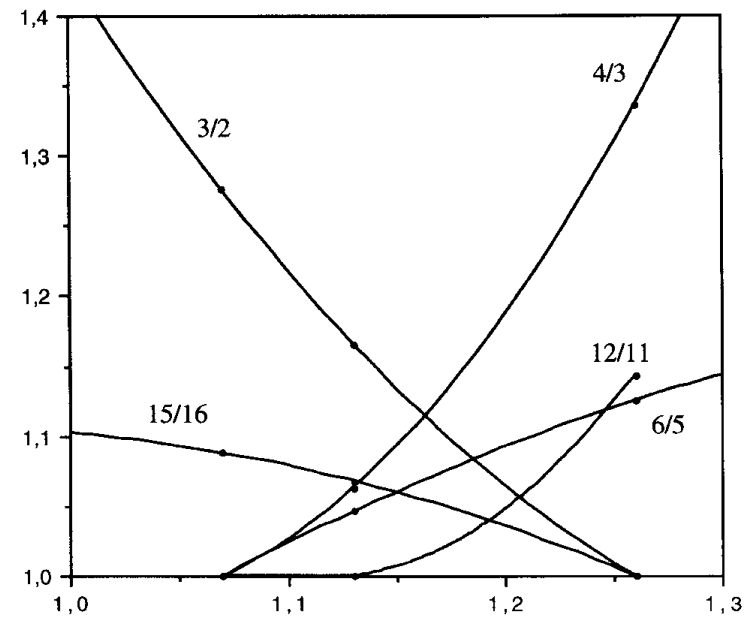

Cis/trans beta-carotene selectivity

Fig. 5. Variation of selectivity for different couples of PAH $v$ s. the cis/trans $\beta$-carotene selectivity (from Ref. 11).

A more precise study of this window diagram indicates that the more efficient $\beta$-carotene selectivity should be close to 1.2 to obtain the separation of all the previous couples of PAH. This value is determined at the crossing of the selectivity curves of the $11 / 12$ and $16 / 15$ compounds, that represents the greatest selectivity which can be reached for the worst two separated couples of PAH (12/11 and 15/16). As the value of 1.2 is set just between the value of the high density bonded silica and the one of the polyfunctional bonded silica, one $25 \mathrm{~cm}$ column of each type have been associated.

A Kromasil column has been selected as high density monofunctional bonded silica and associated with Vydac 201 TP 54 or Hypersil Green PAH. The two other tested polyfunctional phases have not been selected because they inverted the retention order of indeno(1,2,3-c,d) pyrene/benzo ( $g, h, i)$ perylene (15/16) (Figs. 4e, 4f).

The analyses have been done applying an outlet pressure of $10 \mathrm{MPa}$ and a mobile phase gradient during the analysis, increasing the acetonitrile content mixed to the carbon dioxide.

The association of one Kromasil column to a Vydac one is unsatisfactory because compounds 11 and 12 are not fully resolved. This seems to indicate that the polyfunctionality nature of this association is not important enough. However, if two Vydac column are added to the Kromasil column, the whole separation of the $11 / 12$ couple is reached but the $2 / 3$ couple is not well resolved. Thus, the Vydac columns have been changed by the Hypersil Green PAH one.

Working with the association Kromasil-Hypersil Green PAH allows to achieved a sastifactory separation in $27 \mathrm{~min}-$

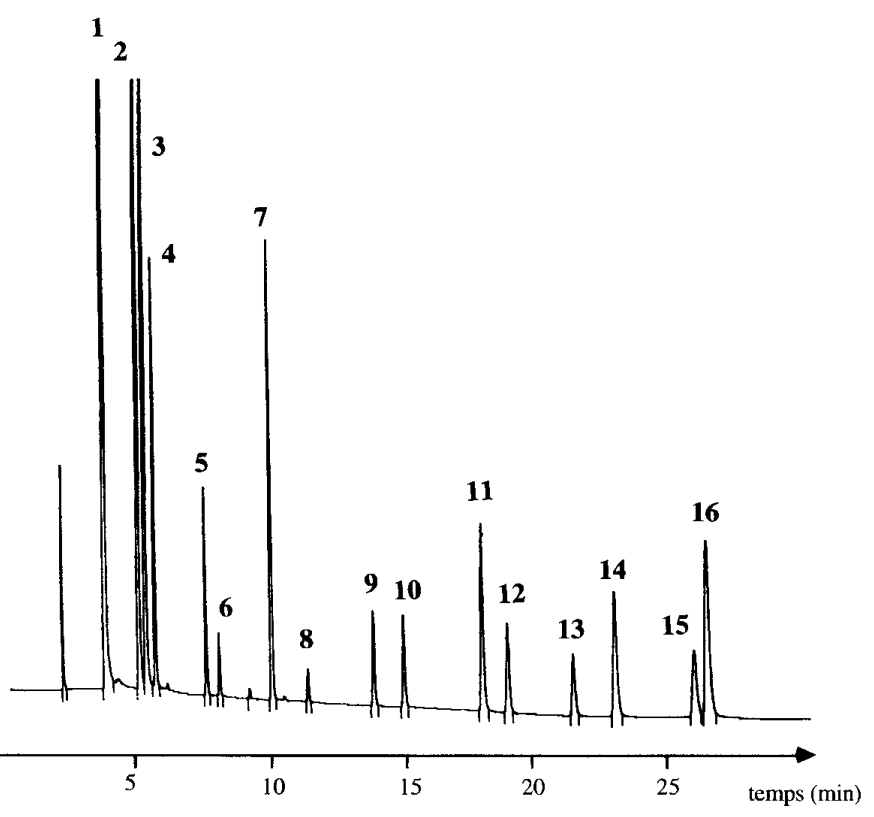

Fig. 6. Analysis of the 16 PAHs by supercritical fluid chromatography. Columns: Kromasil C18 $(250 \times 4.6 \mathrm{~mm})$ - Hypersil Green PAH $(250 \times 4.6 \mathrm{~mm})$. Mobile phase: elution gradient from $\mathrm{CO}_{2} / \mathrm{ACN}(100: 0 ; \mathrm{v} / \mathrm{v})$ to $\mathrm{CO}_{2} / \mathrm{ACN}(50: 50 ; \mathrm{v} / \mathrm{v})$ in 25 minutes, then $\mathrm{CO}_{2} / \mathrm{ACN}(50: 50 ; \mathrm{v} / \mathrm{v})$ during 5 minutes. Total duration 30 minutes. $T=32{ }^{\circ} \mathrm{C}$; Outlet pressure $=10 \mathrm{MPa}$; flow rate $=$ $3 \mathrm{~mL} / \mathrm{min}$; UV detection: $210 \mathrm{~nm}$.

utes (Fig. 6). To increase the separation between acenaphthene and acenaphthylene (2/3), the temperature has been reduced to $32{ }^{\circ} \mathrm{C}$. Consequently, a decrease of selectivity of indeno(1,2,3-c,d)pyrene/benzo $(g, h, i)$ perylene $(15 / 16)$ couple has been obtained. This information latter underlines the proper effect of the analytical temperature that modifies in an opposite way the separation of the two couples of PAHs studied here, when the change of nature of the bonded silica modifies these two separations in the same way.

We can also remark that this association leads to separate an impurity (I) from the fluoranthene. This impurity is at a very low level in a fresh mixture of the 16 PAHs, but it increases as the mixture is ageing.

This supplementary separation is achieved by the Hypersil green PAH column (Fig. 4c).

Important remarks may be made from these results.

The first one is that, as expected, the specific selectivity of each column plays a role in the final separation.

The second one is that the analysis time for the two associated columns is greatly lower than the sum of these obtained with each column alone (Figs. 4b, 4c and Fig. 6).

Two hypotheses may be studied to explain this behavior.

First, coupled two columns increases the internal pressure in the first one, because of the supplementary flow 


\section{Original articles}

transfer resistance induced by the second column. This increases the density of the mobile phase in the first column, that changes the properties of the carbon dioxyde/acetonitrile mixture, i.e, the eluotropic strength should increase. Meanwhile, in part III.1.2, it has been shown that the variation in retention due to an internal pressure modification until $5 \mathrm{MPa}$ was unable to change the retention time of the more retained compounds.

The second hypothesis depends on the use of a mobile phase composition gradient. If the composition gradient is the same using the columns alone, the compounds are introduced in a neat carbon dioxyde mobile phase at the inlet of the column. However, for associated columns, the composition of the mobile phase when the compound arrives in the second column is quite different, mainly for the last compound that enters in the second column 15 to 20 minutes after the injection into the first colum. Thus, taking into account the gradient delay ( 1 minute), and the composition gradient slope (an increase of $10 \%$ of the acetonitrile percentage every 5 minutes), the last compound reaches the second column into a mobile phase composed of 30 to $40 \%$ of acetonitrile. At this moment, the eluotropic strength of the mobile phase is much greater than when the analysis is done using just one column. The time during which the compounds stay in the second column is reduced, that explains the smaller analytical time for the associated column.

The proposed SFC method seems not provide a separation time reduction in comparizon to a HPLC separation. However, the total analytical duration is equal to $35 \mathrm{~min}-$ utes, because only 10 minutes are require for the re-equilibration of the chromatographic system, when it generaly increases twice the analytical time in HPLC. Moreover, the PAH separation by HPLC are often performed with a higher flow rate than the classical one used $(2 \mathrm{~mL} / \mathrm{minutes}$ rather than $1 \mathrm{~mL} /$ minutes), that can reduce the life time of the columns and the efficiency of the HPLC separation. Finally, elution gradient achieved by SFC is a linear one, that allows a greater repeatability.

The analysis of a industrial soil extract, obtained by liquid extraction and sonication, shows four major compounds, that are ligth PAH: naphthalene (3), acenaphthylene (10) acenaphthene (12), fluoranthene (16) and phenanthrene (20) (Fig. 7). This composition seems to be specific of a carbochemistry activity that have led to the soil contamination Numerous additional compounds have been separated and detected, underlining that this separation method developed for PAHs allows the separation of other compounds than those previously studied. With cleaner extracts, the analytical conditions may be changed to reduce the analysis time. Thus, a greater mobile phase flow can be used (from 3 to $5 \mathrm{~mL} /$ minute), increasing the composition gradient slope constant (from 2\%/minute to $3.3 \% /$ minute) for keeping unchanged, as much as possible, the selectivities. Then, analysis time is reduce to 16 minutes and the total analysis including the equilibrium to the initial mobile phase conditions is equal to 24 minutes.

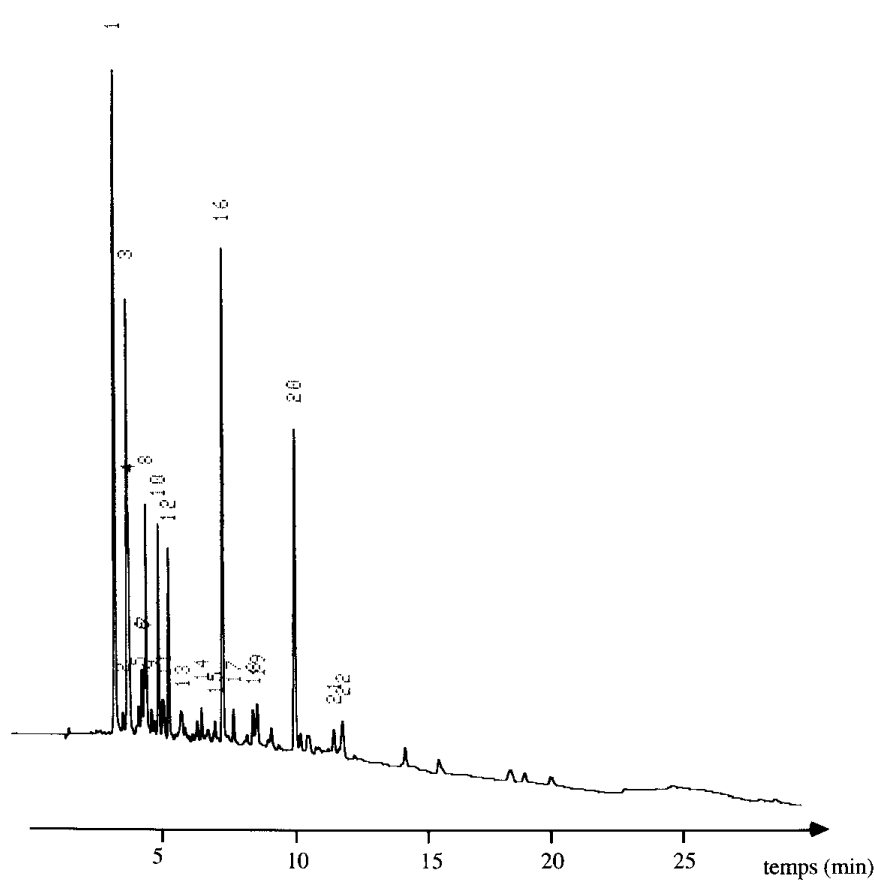

Fig. 7. Analysis of a soil extracts by supercritical fluid chromatography. Compounds: naphtalene (3), acenaphtylene (10) acenaphthene (12), fluoranthene (16), phenanthrene (20).

\section{Conclusion}

Sub/supercritical fluid chromatography is a well-suited separation technique for analysis of complex mixtures, that require both a high selectivity and an important efficiency.

Regarding PAHs separation, the whole separation of the 16 PAHs included in the EPA 610 mixture absolutely requires the association of octadecyl bonded silicas from different nature in functionality. This association is allowed by the properties of the sub/supercritical fluid discharge.

Results prove that the selectivity of each column has its own effect on the final separation. They have also pointed out the modification of retention due to the use of a mobile phase composition gradient with two different bonded silicas.

\section{Acknowledgments}

Following suppliers are thank for their gifts supporting this study: Mrs. Morandini (Life sciences International) and Mr. Polletti (Merck-Prolabo) for the columns, Mr. Hardy and Mr. Cartier (Supelco) for the PAH mixture.

Mr. Hennequin (Wolff environnement, laboratoire de l'Essone) is also thanked for providing the soil extract.

\section{References}

1. Ochmacht, R.; Kele, M.; Matus, Z. Chromatographia 1994, 39, 668 . 
2. Hesselink, W.; Schiffer, R. H. N. A.; Koostra, P. J. Chromatogr. 1995, 697, 165.

3. Wise, S.A.; Bonnett, W. J.; Guentherand, F. R.; May, W. E. J. Chromatogr. Sci. 1981, 19, 457.

4. Sander, L. C. ; Wise, S. A. J. High Res. Chromatogr. Comm. 1988, 11, 383-.

5. Sander, L. C.; Wise, S. A. LC-GC Intl. 1990, 3, 24.

6. Sander, L. C.; Wise, S. A., Lapouyade, R.; Garriques, P. J. Chromatogr. 1990, 524, 111.

7. Kurganov, A.; Unger, K. K.; Eisenbei $\beta$, F. Chromatographia 1994, 39, 175.

8. Kot, A.; Sandra, P.; David, F. J. High Res. Chromatogr. 1994, 17, 277.

9. Heaton, D. M.; Bartle, K. D.; Clifford, A. A.; Myers, P.; King, B. W. Chromatographia 1994, 39, 607.

10. Williams, K. L.; Sander, L. C.; Page, S. H.; Wise, S. A. J. High Res. Chromatogr. 1995, 18, 477.
11. Lesellier, E.; Tchapla, A. in: "Packed Column supercritical fluid chromatography" Berger, C. and Anton, K. Ed., Publ. Marcel Dekker inc, New York 1997; chapter 7, p 195-221.

12. Mourier, P.; Caude, M.; Rosset, R. Analusis 1985, 13.

13. Berger, T. A.; Wilson, W. H. Anal. Chem. 1993, 65, 1451.

14. Sandra, P.; Medvedovici, A.; Kot, A.; David, F. in "Packed Column supercritical fluid chromatography" Berger, C. and Anton, K. Ed., Publ. Marcel Dekker inc, New York 1997; chapter 6, p 161-194.

15. Lesellier, E.; Krstulovic, A. M.; Tchapla, A. Chromatographia 1993, 36, 275.

16. Lesellier, E.; Tchapla, A. Proceedings of the $3^{\text {th }}$ conference on the supercritical fluid, Grasse, France, 1996, p 115.

17. Lesellier, E.; Tchapla, A.; Subra, P.; Tufeu, R. Proceedings of the $3^{\text {th }}$ conference on the supercritical fluid, Grasse, France, 1996, p 257. 